\title{
Enhancing Education and Learning Capabilities via the Implementation of Video-to-Video Communications
}

\author{
Ioannis P. Chochliouros ${ }^{1}$, Anastasia S. Spiliopoulou ${ }^{2}$, Evangelos Sfakianakis ${ }^{1}$, \\ Ioannis M. Stephanakis ${ }^{2}$, Donal Morris ${ }^{3}$, and Martin Kennedy ${ }^{4}$ \\ ${ }^{1}$ Research Programs Section, \\ Hellenic Telecommunications Organization (OTE) S.A., \\ 99 Kifissias Avenue, GR-151 24, Athens, Greece \\ \{ichochliouros, esfak\}@oteresearch.gr \\ ${ }^{2}$ Hellenic Telecommunications Organization (OTE) S.A., \\ 99 Kifissias Avenue, GR-151 24, Athens, Greece \\ \{aspiliopoul, estephan\}@ote.gr \\ ${ }^{3}$ RedZinc Services Ltd., \\ Taylor's Lane, Dublin 8, Dublin, Ireland \\ dmorrisaredzinc.net \\ ${ }^{4}$ Scoil Naisiunta Cholmcille (Skryne), \\ Skryne, Tara, Co. Meath, Ireland \\ skryne.n.s@gmail.com
}

\begin{abstract}
The LiveCity Project addresses a number of communities where the citizens of a city can have specific challenges and derive immediate benefits or advantages from the proper and effective use of live interactive high definition video-to-video $(\mathrm{v} 2 \mathrm{v})$ communications over the Internet, for various sectors of applications and/or related services. One of these communities is the educational community, where major benefits and impacts may raise, as a result of the innovative $\mathrm{v} 2 \mathrm{v}$ implementation and use, to support teaching and learning activities. In this paper, we propose a specific context to satisfy this challenge, based on the formation of a "network" of involved schools, where we also identify essential users' needs and propose distinct educational use cases (i.e.: $\mathrm{v} 2 \mathrm{v}$ for school-to-school communication and $\mathrm{v} 2 \mathrm{v}$ for an interactive homework channel). In addition, we discuss potential benefits from this action and we identify legal and ethical issues that may affect further progress.
\end{abstract}

Keywords: Digital economy, eLearning, Internet, information and communication technologies (ICTs), video-to-video (v2v).

\section{Introduction}

Information and communication technologies (ICTs) can enable learning and education activities and help people pick up new skills in the modern digital-based economies and societies [1]. Further than their broad mission to serve society as a whole, education and training systems are also of specific importance in helping to perform sustainable growth and development in a variety of sectors. The 
modernization of existing European education and training systems via the effective implementation and use of innovative communication methods and related technologies or services can provide adequate responses to the demands of the knowledge-based society. Continuous and wireless access to digital resources is redefining the ways in which we perceive and use our dimensions of social time and space. In particular, the proper inclusion of new, open and flexible ICTs have the potential to significantly advance the progress to Europe's future prosperity and social cohesion. The role of traditional educational institutions and schools in educating younger generations has never been more important than it is today [2]. To succeed in tomorrow's digital challenges, pupils and students need to be equipped with the knowledge and skills essential to deal with constant change in their private and professional lives. They require not only the technical skills necessary to engage with the new technologies - so-called "digital literacy" - but also the "softer" skills such as creativity, problem-solving and team work. In this scope, the defined boundaries of learning are constantly changing [3]. Technological developments, such as the Internet, mobile communications and virtual environments, generate remarkable options to sustain learning in new and quite effective and productive ways. These are opening up new opportunities for learning, for example by allowing the learner to be mobile, connected, and digitally equipped [4].

\section{Context for a Collaborative Educational Development}

Following to the previous challenges, the LiveCity ("Live Video-to-Video Supporting Interactive City Infrastructures”) Project (Grant Agreement No.297291), directly focuses to a modern environment world where live high-definition (HD) interactive video-to-video (v2v) can be as easily available on any device on any city street, as any other actual communication facility. The effective inclusion of (interactive) video communication can thus enhance the way of citizens' every-day communications and of their vivid inclusion in the modern societies. The initiative becomes particularly innovative as it intends to propose the pilot development of a number of v2v-based services initially in four cities for a diverse range of applications in the areas of Municipal Services, City Education, City Experiences, Emergency Services and eHealth. The "LiveCity" will "pilot" these live high quality video-to-video applications in order to evaluate social utility and to demonstrate real and immediate value to city citizens.

Among the already indentified LiveCity-based priorities is also the detailed perspective for supporting educational activities via the inclusion of $\mathrm{v} 2 \mathrm{v}$ services in schools. In particular, two scenarios are to be deployed proposing the implementation of live interactive $\mathrm{v} 2 \mathrm{v}$ communications for use in schools and museums/cultural institutes. Teachers are seeking to enhance learning experiences by using v2v. Ten schools in Dublin and two schools in Athens will experiment with interactive and collaborative learning basis, using sharing educational experiences, while city educational providers will study pedagogical outcomes.

The museum exhibit will allow for visitors at two locations in Athens (OTE's Telecommunications Museum) and Luxembourg (Cité Bibliothèque) to interact 
within one another in a "joint experience". The aim is to promote awareness of each city to visitors at both locations. The final design will use live interactive v $2 \mathrm{v}$ as a basis for the interaction and will, in addition, use a projection screen and/or multitouch technologies and, where appropriate, mobile devices. Here, the essential aim is to encourage greater collaboration, figure and knowledge sharing between city dwellers, visitors and public spaces, for example museums, cultural institutes and galleries. The "school element" will focus on interconnecting 10 schools centered around Dublin and implementing a range of collaborative projects. A wide selection of themes (e.g., literacy, sports, arts, maths) are available and the most promising of these will be structured into a range of collaborative learning projects between the schools, following discussions with the involved entities and pedagogical. In addition, within this education-oriented activity, two Greek schools from the Municipality of Vrilissia-Athens are also to be connected to the OTE's Telecommunications Museum and will participate to specific educational programs -promoted by the museum itselfas advanced users, by also providing feedback. The connection of the schools will include the installment of a screen for a certain period at the schools premises, providing some content from the museum. Participation will mostly include viewing of the collaborative effort between the museum and interacting, where possible. The main aim is to support the development of a collaborative environment, allowing online collaboration between two or more locations using interactive live $v 2 \mathrm{v}$ in combination with augmented reality. This will encourage greater collaboration, learning and knowledge sharing between city schools.

LiveCity intends to integrate the various required technologies to provide a plug-in capable of supporting two-way, multi-site and live v2v that allows interactive content to be embedded in the video stream. This will take the form of each site having a large projector screen, upon which an interactive video stream is available as well as an interactive table top interface that supports tracking of real-world objects coupled with augmented reality technologies that allow the addition of interactive content to real-world objects.

\subsection{Establishing a Network of Schools}

As ICT becomes more and more prevalent within schools, LiveCity proposes to pilot a scenario where a video-to-video network is established between city schools to enhance education. The explosion of smart phones and the anticipated explosion of tablet devices with low cost high-definition cameras and the deployment of $4 \mathrm{G}$, supports the option of $\mathrm{v} 2 \mathrm{v}$ to becoming a reality for the mass market, also including the educational and teaching community. Thus, LiveCity will build a collaborative video experience for 10 schools in Dublin city, anchored around Marino Institute of Education and one of the schools, called Scoil Naisiunta Cholmcille (the "Skryne School"), that will be the Lead School. LiveCity will make use of school networks infrastructures (e.g., Education Centers, Teacher Professional Networks) for disseminating information about the LiveCity capabilities to the educational community, ask for schools' participation and sharing educational experiences. Each school will be represented by a teacher from the school in the local community that 
will provide practical day to day inputs. From a network engineering point of view there is limited value in having just one school. It is important to test as many sites and with as many end-users as possible in order to implement, demonstrate and validate the project's approach, to maximize reachability. More users participating will enhance the robustness by giving feedback and allowing the engineers to fill any gaps identified. From a user point of view more users leads to diversity of requirements and perspectives. Greater diversity will lead to a better understanding of how to achieve maximum utility from the v2v service for educational purposes.

A specific Stakeholder Committee will be established to guide the pilot and provide a perspective grounded in the local user community. The committee will include a representative from the lead school, a teacher, a parent, a representative of the local town council and a representative from Marino Institute of Education. This committee will "liaise" with the participants from the 10 selected schools.

\subsection{Defining User Needs and Creating Education Use Cases}

LiveCity will implement at least two educational use cases using the Future Internet capabilities to realise $\mathrm{v} 2 \mathrm{v}$ in the involved schools. In addition, user scenarios are to be defined in parallel with any related users needs, while there will be effort to suggest ways of exploitation of the developed tools by the education community. Use cases of the one-to-one (e.g., school-to-school) and of the one-to-many type will be explored, so as to investigate both types of communication capabilities. In particular, two use cases are to be implemented and these are relevant to: (i) The use of v2v in a schoolto-school (S2S) communication, and; (ii) the use of $\mathrm{v} 2 \mathrm{v}$ in a Homework Channel (HW). Both are actually considered as challenging and promising".

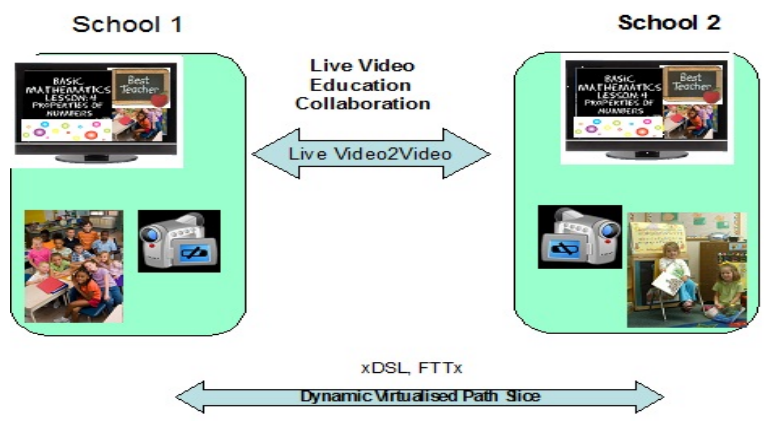

Fig. 1. School-to-School video interaction

In the former use case, video-to-video is to be used between schools, for applications such as projects, culture awareness and other topics (as shown in Fig.1). Themes related to learning of literacy and arts along with other educational activities, can also be implemented with v2v. Schools should be encouraged for communicating and displaying their work to others. In the latter use case, v2v is also to be used between pupils-students after school from their home to a central educational support 
provider (as shown in Fig.2). The purpose is to assist and enhance homework activities. An authentication system will be included with the homework channel to support privacy. Extensive technical and usability testing and reporting will be conducted following the implementation of the selected uses cases to schools. The analysis will be based on questionnaires, as well as ad-hoc research activities.

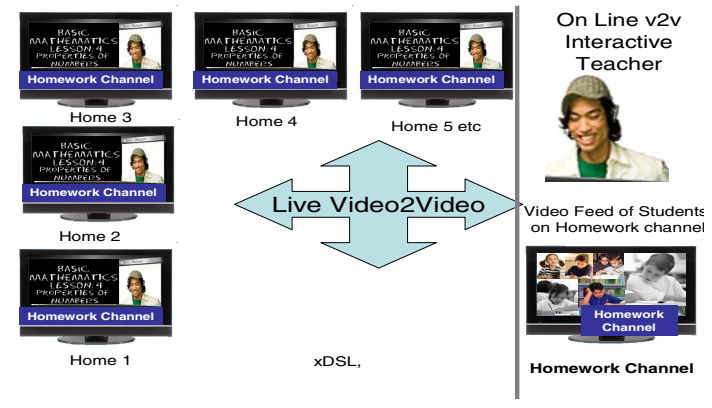

Fig. 2. Interactive Homework Channel

User scenarios to be explored include: (i) The use of $v 2 v$ in a literacy education scenario ([5], [6]), and; (ii) the use of v2 $\mathrm{v}$ in a sport education scenario.

In the first case we can distinguish two potential dimensions: (a) Firstly we propose that a local piece of historical folklore or legend is researched and stuctured based on the relevant curriculum objectives. This can be presented both in the great tradition of story-telling and as a dramatic piece interpreted by the pupils. This facilitates integration across the subject areas as pupils research, edit, prepare and produce the finished article. Perhaps an on-line book may be the final product or maybe even a mini dramatic piece scripted by the pupils. (b) For the second strand of this area, we propose two scenarios: (1) The collaborative writing of a story by a group of remote participants. For instance, a group of pupils (e.g., 10-11 year olds) can be divided into groups of 3-4 participants each, and directed to compile and agree on the outline of a story. Then, each group is to be tasked with writing a page or a chapter or a scene of the story, before passing on to the next remote group. The story can develop in a reciprocal cycle as the pupils see fit and agree. The pedagogical experience can be further enhanced by editing conferences for all participants, heightening their critical analysis skills. In this manner, all participants should be able to develop ownership of the "finished product" which may be presented again on-line to remote participating schools. (2) As an alternative, pupils may be invited to describe their own experience of living and school-life in their own particular area. Others can do the same and compare, eventually through the link. Written pieces can then be submitted for all to study. At a later stage, evaluations can be made by the pupils on the differences and similarities which exist for them all.

In the second case, $v 2 \mathrm{v}$ could be used in a sport-oriented education scenario. This module is inspired by "sport as a unifying force across the world". Students will be invited to tackle a set of drills in a universally played sport (e.g., basketball) to improve their skills. An initial set of goals or challenges are to be defined to establish 
base-line information on the skill level of the participants. Then, after a period of practice, progress will be assessed and the results are to be measured in a series of time or target set exercises. All participating schools will undertake the same practice and undergo the same test regime. Results may then be compared and analysed. In this scope, video evidence will be used to identify reasons for any spread in summative results, for example; Instructional factors, equipment, prior knowledge, weather, poor facilities, basic skill set missing, etc. Suggestions will emanate as to how skill set and performance can be further improved. This will be measured on an on-going basis. In a complimentary context, pupils may have the opportunity to showcase a sport popular in their area but not widely known to others. For instance, it may be Hurling, Olympic Handball, Netball, Pelota, or other sport relevant to the schools involved. Rules and regulation may be relayed to partner schools and practical demonstrations could also be offered. Instruction on how to play or train may be exchanged. Challenges may be set for those wishing to engage and after an agreed time scale measurement of success may be taken. This interchange of ideas would prove most beneficial to all. It should be noted that this use case maybe complicated to implement, as the capability of video capture will be significantly less then available as on a "professional TV soccer game". Nevertheless, schools can experiment in this area and see what is possible, with low cost cameras-tablet devices.

A similar activity might also be performed, specifically focused on arts. In dealing with the subject area of Arts, a twin-pronged approach might be taken into account: We are suggesting that students tackle the study of a particular artist (for example: Miro, Picasso or Van Gogh), or a period (for example: impressionism, cubism, etc.), and then report their findings via video link. This could be either live or in minidocumentary format. In the latter, the pupils will also learn editorial and presentation skills. A degree of teacher input may determine whether one group concentrates on earlier work and influences later offerings. Students could also be invited to recreate samples of the work they study through digital or true-to-life representations. The second strand would incorporate the creation of a piece of art work for display to others. A potential "candidate" could be a mosaic based on local or agreed folklore (say perhaps, zoned on a Dutch/Spanish/French/Irish legend or prominent historical figure). Each country has its own values to cherish and advertise to the other participants. Students can exchange ideas both practical and creative, as the project develops. Advice on techniques and on content can help to share and advance.

\subsection{Expected Benefits}

The development of the educational activities, as presented in the previous section, may implicate explict and/or implicit benefits, covering a variety of domains. The use of the LiveCity-based $v 2 v$ application can be innovative in the context of the educational technology, as it is expected to create new dimension and to harass new opportunities and options for the students/pupil and the teachning community. In addition, it can further enhance educational awareness -even at a wider or panEuropean level- in arts, literacy and sports, by connecting schools with appropriate video-to-video links. 
It also implicates remarkable societal impacts including, for example and among others: (i) The ability of schools -potentially across Europe- to collaborate on projects, therefore allowing for the sharing of resources and teachers; (ii) The ability for leading teachers to provide classes to students across the globe; (iii) Provision of teachers' training facilities between schools and training centers; (iv) Improved cultural exchanges between schools from across Europe, increasing awareness of arts, science and social issues, inter-alia; (v) Increase of parental involvement in education through the provision of teacher-parent training, therefore lowering the gap of knowledge between parents and their children; (vi) Ability to create richer educational experiences using interactive v2v that will improve pupil attainment; (vii) Contribute towards improving the learning experience of pupils; (viii) Better transfer the skills offered in better schools towards the "less well performing" schools between pupils and between teachers, in a cost-efficient way; (ix) Facilitate EU-level and national political ambitions to improve education standards and, eventually, to create an EUwide universal education system accessible by all sections of society; (x) Gain of knowledge through access to expert teachers, and; (xi) Contribute to generate new knowledge, new ideas for learning and teaching through the use of video archives.

Furthermore, this involves potential economic impacts as, for example: (i) Ability to "share teachers and resources" across locations, thus resulting to lowering of costs; (ii) Early identification of educational scenarios to allow for development post-project of education services; (iii) Ability to increase the number of teachers and pupils who can undertake training, therefore lowering the cost of providing training on a "perperson" basis. The v2v inclusion is an innovative concept which, apart from capitalizing on existing technologies and infrastructures, also tries to provide several social services also including education ${ }^{1}$. These services are considered under an international scope which can provide comparative results between the different countries' approaches for them and promote their use to more communities.

\section{$3 \quad$ Ethical and Legal Context}

The intended educational oriented LiveCity effort involves the delivery of services to participants, some of whom are likely to be children below the age of consent. Following to the requirements implied by the actual European regulation ([7]), the research activities carried out under any Seventh Framework Project (FP7) -also including the scope of the LiveCity effort- will be in full compliance with fundamental ethical principles. Consequently, LiveCity will abide by all applicable and any future EU [8] and national legislations, as well as by the relevant international guidelines. Ethical issues and/or applications as appropriate will be taken into account by the designated Stakeholders' Committee to evaluate the related pilot that is to be focused to the educational scenario. The proposed approach and implementation will adhere to the laws, regulations and ethical guidelines of the countries where the research is carried out (i.e.: Ireland and Greece). Other ethical issues, if any, will be properly addressed to oversee and safeguard adherence to existing ethical standards and practices.

\footnotetext{
${ }^{1}$ Other activities of the LiveCity Project effort may focus on municipal and eHealth services.
} 
The Project recognizes the vulnerability of children [9], their special place in EU external actions [10] and the EU commitment to respect under international and European Treaties the United Nations (UN) Convention on the Rights of the Child $(\mathrm{CRC})^{2}$. In view of the possibility of such situations, LiveCity will in particular consider Article 12 of the CRC when designing consent forms and seeking consent from participants who are children, and their parents, guardians or "legal representatives". Beneficiaries shall assure to each participating child who is capable of forming her/his own view the right to express those views freely in all matters affecting her/him, and her/his views shall be given due weight in accordance with the age and maturity of the child. LiveCity recognizes that informed consent of parents or legal representatives (the definition of "legal representative" is to be considered in accordance with the legislation of the host country) must be obtained, but also that when the child is able to give assent such assent should additionally be obtained. In the same scope, a child's refusal to participate or to continue to participate will be acted upon and the child, if already in the study, will be immediately withdrawn.

Interviews with pupils/students and their families can also be conducted. These will follow best practice with respect to the provision of adequate information to make a decision, informed consent, anonymity and storage of data, freedom not to participate or withdraw from an interview at any point. Similar measures will be taken with respect to any other potential interviews that might be carried out with the involvement of local or national authorities.

The processes and documentation for obtaining consent will be comprehensive and separate for parents/legal representative and for children. Project information and consent forms will be designed so that any participant is fully informed about the study and participant-safety safeguards, and able to make a free and informed decision. Information sheets will be designed in accordance with the age of the children. Indicative information to be contained on information sheet for obtaining written consent may indicatively include the following: (i) Statement that the Project involves of research subjects; (ii) Explanation of purpose of the research; (iii) Expected duration of the subject's participation; (iv) Description of procedures to be followed; (v) Statement that participation is voluntary; (vi) Information about who is organizing and funding the research; (vii) Description of any foreseeable risk, discomfort or disadvantages; (viii) Description of benefits to the subject or to others which may reasonably be expected from the research (no inappropriate expectations); (ix) Disclosure of appropriate alternative procedures for treatment that might be advantageous to the subject. (x) Statement describing procedures for ensuring data protection, confidentiality and privacy; including storage of personal data, usage of data, access of data, and destruction of data; (xi) Explanation as to whether there are any treatments or compensation if injuries or complications occur, and if so, what they consist of, or where further information may be obtained; (xii) Contact references for answering questions about the research and the subject's rights; (xiii) Statement offering the subject the opportunity to ask question and withdraw at any

\footnotetext{
${ }^{2}$ Convention on the Rights of the Child. [http: / /www2 ohchr.org/english/law/crc.htm]
} 
time from the research and without consequences; (xiv) Explanation of what will happen to the data at the end of the research, and; (xv) Information on what will happen to the results of the research.

Ethical Issues are very important from the point of view of involvement of the $\mathrm{v} 2 \mathrm{v}$ with children. A consent declaration will be obtained for any family whose children are involved in the schools pilot. The v2v interaction will be in a private domain and issues such as security and confidentiality are to be adequately addressed. LiveCity is to be performed by industry and educational professionals aimed at sharing best practice for teachers and broadening the learning experience for the participant children. Parents and/or legal representatives will be asked to provide written consent allowing the classroom recordings and their children's written work to be used as part of the project effort. Children's inclusion and their recorded images and sounds in project is -and will remain- voluntary. From the teachers' point of view it is estimated that children's participation will have considerable benefits for them, both from a learning and a self-esteem aspect. Records that are to be shared and used are: (i) Audios and videos of class meetings and displays: These are important to give a solid base to shared content. (ii) Copies of written work: This will be an important source of information to illustrate how each topic area develops to presentation stage. (iii) Photographic records: Photos/video of children and adults engaged in class activities may be used for sharing with fellow partners. To safeguard confidentiality, children's surname will be removed from all the documents that will be used for the project purposes. It is important to note, however, that in instances where video/audio segments or handwritten samples of children's work are included in a multimedia presentation, it is possible that someone who knows children may correctly identify them. If consent is granted for the collected recordings and documents to be used for the educational purposes of the project, then children's written work and the recordings in which he/she can be seen and heard may be used in relation to all aspects of the project. If consent is withheld, then children's written work will not be used and audio/video segments where a child can be identified will not be used.

\section{Synopsis}

Open and flexible forms of technology-enhanced learning contribute more and more to the quality of education and training systems. ICTs make teaching and training processes more tailored to the needs of the learner conformant to his specific profile, help advance and support innovation in pedagogy, and make learning more engaging, for all potentially involved actors. They also sustain organisational transformation within education and training institutions, which will help to improve educational quality, and to extend access to learning beyond traditional educational settings. In this context, eLearning is progressing from the basic use of ICT for learning (e.g. as a research tool and replacement for books), to new forms of education and training which emphasise creativity and collaboration - and new skill requirements for the knowledge society. The LiveCity effort intends to provide a systematic user co-creation approach, integrating technology to deliver new innovative processes in 
selected domains, also including teaching and educational activities, via the effective implementation of live high quality video-to-video communications over the Internet. In the scope of the present paper we have discussed, in particular, the context for developing a collaborative and interactive educational development mainly via the establishment of a network of schools. In addition we have defined some essential users' needs, that is by identifying as "end-users" the involved pupils-students and their teachers, and we have proposed some potential educational use cases, where the use of $v 2 \mathrm{v}$ can be considered between schools or for the proper operation of an interactive homework channel. The paper has also identified some potential sectors of interest, for instance related to literacy, sports and arts, where v2v communications could be implemented and used in order to support learning activities, on the one hand, and to provide motivation for dealing with additional concepts, on the other, always with the aim of enhancing and extending children's knowledge. Based on the actual scope of the LiveCity context, we have estimated, qualitatively, several essential benefits that might be realized via the proposed innovative action. Furthermore, we have focused on and discussed some legal issues together with necessary ethical principles, practices and requirements that have to be taken into account, while proceeding to the real implementation of the proposed scenarios or use cases.

\section{References}

1. European Commission, Information Society and Media: Education and Information Society: Linking European Policies. Luxembourg (2006)

2. Bransford, J.D., Brown, A.L., Cocking, R.R.: How People Learn: Brain, Mind, Experience and School. National Academy Press, Washington, DC (1999)

3. Commission of the European Communities: Communication on "i2010 - A European Information for Growth \& Development” [COM(2005)229 final, 01.06.2005]. European Commission, Brussels (2005)

4. Rogers, Y., Price, S.: Using Ubiquitous Computing to Extend and Enhance Learning Experiences. In: van't Hooft, M., Swan, K. (eds.) Ubiquitous Computing in Education: Invisible Technology, Visible Impact, pp. 329-347. Lawrence Erlbaum Associates Inc., Mahwah (2007)

5. European Commission: Digital Literacy - European Commission Working Paper and Recommendations from Digital Literacy High Level Expert Group. European Commission, Brussels (2010), http: / / ec.europa.eu/information_society/ eeurope/i2010/digital_literacy/index_en.html

6. Dolton, P.: What Do Policy Makers Need to Know about the Skills of Young People and the School to Work Transition? OECD, Paris (2004), http://www.oecd.org/ dataoecd/48/50/34474626.pdf

7. European Parliament and Council: Decision No.1982/2006/EC of 18 December 2006 concerning the Seventh Framework Programme of the European Community for research, technological development and demonstration activities (2007-2013). European Parliament and Council (Official Journal (OJ) 30 December 2006, No.L412, pp. 1-41), Brussels (2006) 
8. European Parliament and Council: Directive 95/46/EC on the protection of individuals with regards to the processing of personal data and on the free movement of such data (OJ 23 November 1995, No L.281, pp. 31-50). European Parliament and Council, Brussels (1995)

9. European Commission: Ethics in Research and International Cooperation - Preamble. Ethical Review in FP7. Research Directorate, General Directorate L, Science, Economy and Society, Unit L3, Governance and Ethics (2007), ftp: / / ftp. cordis . europa.eu/pub/fp7 / docs / developing-countries_en.pdf

10. Commission of the European Communities: Communication on "A Special Place for Children in EU External Action". Communication [COM(2008)55 final, 05.02.2008]. European Commission, Brussels (2008) 